\title{
Ciclo de Mejora Docente para la asignatura de Diseño y Aplicaciones Multimedia del Grado en Comunicación Audiovisual
}

\author{
ANTONIO GÓMEZ-AGUILAR \\ Universidad de Sevilla \\ Departamento de Comunicación \\ Audiovisual y Publicidad \\ agomez16@us.es \\ ORCID: https://orcid.org/0000-0003-2819-3441 \\ D.O.I.: http://dx.doi.org/10.12795/JDU.2018.i01.57 \\ Pp.: 1016-1034
}

\section{Resumen}

Experiencia de diseño, realización y autoevaluación de un ciclo de mejora de 12 horas para la asignatura de Diseño y Aplicaciones Multimedia del Grado en Comunicación Audiovisual en la Universidad de Sevilla durante el curso 2018/2019.

Palabras clave: Diseño y aplicaciones multimedia, Comunicación Audiovisual, docencia universitaria, experimentación docente universitaria 


\section{Breve descripción del contexto}

La comunicación se centra en el 2o Ciclo de Mejora que he realizado para mi asignatura de "Diseño y Aplicaciones Multimedia" dentro del Curso General De Docencia Universitaria perteneciente al Programa de Formación e Innovación Docente del Profesorado 2018 - Fase Preliminar. "Diseño y Aplicaciones Multimedia" es una asignatura obligatoria del cuarto curso del Grado en Comunicación Audiovisual y del Doble Grado en Comunicación Audiovisual y Periodismo. En la clase hay 67 alumnos de dos grados; 65 del Grado en Comunicación Audiovisual y 2 del Doble Grado en Comunicación Audiovisual y Periodismo. La asignatura tiene 3 créditos teóricos y 3 créditos prácticos.

La adaptación de los contenidos de este ciclo está motivada por la iniciativa de los alumnos, queremos que nuestro proyecto sea una experiencia educativa en nuesros alumnos (Vergara, 2017); de esta forma, partiendo de los proyectos de cada grupo, ir aportando soluciones desde la teoría a sus desafíos en el desarrollo de sus proyectos. Guiando los proyectos iremos cubriendo fases en su realización y aportando los conceptos necesarios. El contenido que hemos seleccionado para este ciclo de mejora es el bloque de "Especificaciones Funcionales de un proyecto transmedia". En este bloque hay clases en aulas teóricas y clases en aulas prácticas. Todas las aulas disponen de cañón, ordenador PC y pizarra. Las aulas prácticas son aulas informáticas con equipos Mac con el paquete de Adobe CC con el trabajaremos en las prácticas de InDesign.

\section{Diseño previo del Ciclo de Mejora Docente}

El mapa de contenidos recoge los contenidos fundamentales del tema que se desarrollarán durante el ciclo de mejora. En él reflejamos las diferentes unidades y bloques de contenidos, así como las relaciones que se establecen entre ellos. También hemos señalado el tipo de contenido 
según la siguiente codificación: $(\mathrm{C})$ = Contenidos Conceptuales; $(\mathrm{PI})$ = Contenidos Procedimentales Intelectuales; $(\mathrm{A})$ = Contenidos Actitudinales; $\mathrm{y}(\mathrm{P})=$ Contenidos Prácticos.

El tema es una fase dentro del desarrollo de un proyecto. Dicho proyecto se asienta sobre una serie de contenidos conceptuales que nos permiten luego desarrollar el proyecto a través del análisis, la creación de modelos, la categorización, las relaciones entre conceptos y la visión crítica (PI). En esta parte haremos alguna práctica informática $(P)$ perteneciente al proyecto. Los contenidos actitudinales (A) están presentes desde la concepción y diseño del proyecto hasta los eventos claves en los que se materializa

\section{TEMA: Especificaciones Funcionales del proyecto transmedia}

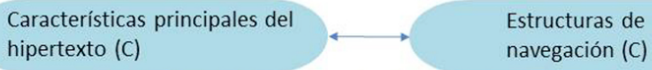

Estructuras de hipertexto (C) navegación (C)

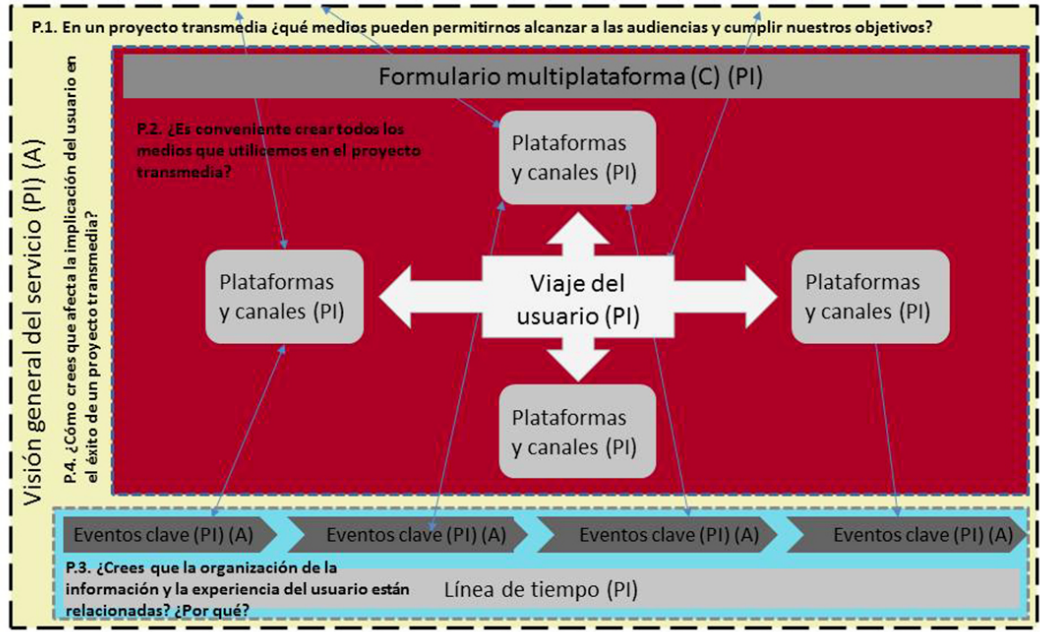

Figura 1. Mapa de contenidos

Hay cuatro problemas con los que pretendemos "crear un entorno para el aprendizaje crítico natural" (Bain, 2007) y en torno a los que giran los contenidos del tema (P.1.) (P.2.) (P.3.) (P.4.) que hemos señalado con colores distintos. El P.1. y el P.4. son problemas generales que afectan al diseño global del proyecto; P.2. es un problema concreto

Jornadas de Formación e Innovación Docente del Profesorado | № 1 (2018)

Esta obra se distribuye con la licencia Creative Commons Reconocimiento-NoComercial-SinObraDerivada Internacional (CC BY-NC-ND 4.0.) 
sobre las Plataformas y Canales que vamos a usar en el proyecto y P.3. es un problema concreto sobre la experiencia del usuario a lo largo del proyecto y está asociado con los eventos y la línea de tiempo de los mismos.

Hemos establecido dos Modelos Metodológicos; uno para la primera sesión y otro para el resto de sesiones.
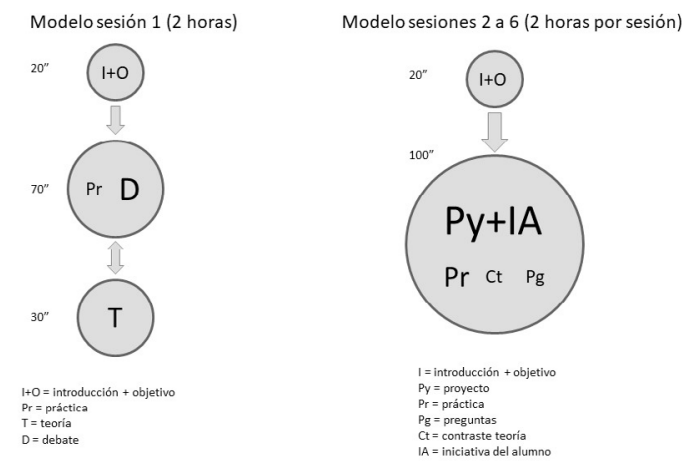

Figura 2. Modelos Metodológicos

Dentro de las sesiones, para cada actividad que hemos planteado hemos realizado una ficha detallada:

\begin{tabular}{|l|l|l|}
\hline Número ficha: Sesión $1 /$ Ficha 1 & Fase metodológica: $1+0$ & Duración: 20" \\
\hline
\end{tabular}

Introducción y objetivos de la sesión: al principio de la sesión voy a realizar una introducción de manera informal sobre el contenido de esa sesión. En esa introducción voy a fijar los objetivos de la sesión que serán explicar los conceptos relacionados con las Especificaciones Funcionales de nuestros proyectos transmedia.

Material necesario: PPT

Número ficha: Sesión 1/ Ficha 2

Fase metodológica: $\mathrm{Pr}+\mathrm{D}$

Duración: $70 "$

Jornadas de Formación e Innovación Docente del Profesorado I № 1 (2018) Esta obra se distribuye con la licencia Creative Commons Reconocimiento-NoComercial-SinObraDerivada 
Práctica de visionado: para explicar los conceptos relacionados con las Especificaciones Funcionales de nuestros proyectos transmedia vamos a ver un video que analiza el caso práctico de un proyecto concreto, "El universo transmedia de la serie La Peste". El visionado del vídeo va acompañado de una hoja de visionado donde los alumnos irán anotando, sobre un esquema dado, algunas de las ideas que se apuntan en el vídeo para el debate posterior. El objetivo del debate es ir desarrollando las ideas teóricas que se plantean en el vídeo e ir enlazándolas con los contenidos de nuestro tema.

Material necesario: PPT, Vídeo "Universo transmedia de La Peste", hoja de análisis para el debate.

\begin{tabular}{|l|l}
\hline Número ficha: Sesión 1/ Ficha 3 & Fase metodológica: $T+D$ \\
\hline
\end{tabular}

Duración: 30"

Teoria: Al finalizar el debate en una presentación en PowerPoint iremos concretando esos conceptos teóricos y sistematizando la información para los alumnos.

Material necesario: PPT.

\begin{tabular}{|l|l|}
\hline Número ficha: Sesión 2/ Ficha 4 & Fase metodológica: I + 0 \\
\hline
\end{tabular}

Duración: $20 "$

Introducción y objetivos: en esta introducción revisaremos rápidamente dónde nos quedamos el día anterior y fijaremos los objetivos de la sesión que serán diseñar, por grupos, las Especificaciones Funcionales de cada proyecto práctico de la asignatura.

Material necesario: PPT.

Número ficha: Sesión 2/ Ficha 5

Fase metodológica: $P y+$

$\mathrm{IA}+\mathrm{Pr}+\mathrm{Ct}+\mathrm{Pg}$

Duración: 100"

Proyecto + Iniciativa de los Alumnos + Contraste de la Teoría + Preguntas: el resto de la sesión será de trabajo en grupo. Sobre una serie de fichas del proyecto que se pasarán a los grupos, los alumnos deben definir antes de acabar la clase un primer borrador de las Especificaciones Funcionales de su proyecto.

En ese tiempo el profesor estará pasando entre los grupos comentando sus ideas. En este tiempo, en función de la iniciativa de los alumnos en torno a cada proyecto, se contratarán conceptos teóricos y se resolverán dudas que no hayan sido expuestas anteriormente. Al final de la clase cada grupo entregará sus fichas de proyecto.

Material necesario: PPT y fichas de proyecto [plantillas 4 a 11 en Enseñanza Virtual].

\begin{tabular}{|l|l|l|}
\hline Número ficha: Sesión 3/ Ficha 6 & Fase metodológica: I + O & Duración: \\
\hline
\end{tabular} $20 "$ 
Introducción y objetivos: en esta introducción revisaremos rápidamente dónde nos quedamos el día anterior y fijaremos los objetivos de la sesión que serán diseñar, por grupos, el producto multimedia que van a realizar dentro de cada proyecto práctico de la asignatura.

Material necesario: PPT.

Número ficha: Sesión 3/ Ficha 7

Fase metodológica: $P y+$

$\mathrm{IA}+\mathrm{Pr}+\mathrm{Ct}+\mathrm{Pg}$
Duración:

$120 "$

Proyecto + Iniciativa de los Alumnos + Contraste de la Teoría + Preguntas: esta sesión será de trabajo en grupo con ordenadores. Sobre las fichas del proyecto del borrador de las Especificaciones Funcionales de su proyecto los alumnos deben concretar que parte práctica [producto multimedia] van a realizar. Este trabajo incluye la definición del producto multimedia y un ejercicio práctico [01/Creación de un documento interactivo] con el software Adobe InDesign.

En ese tiempo el profesor estará pasando entre los grupos comentando sus ideas. En este tiempo, en función de la iniciativa de los alumnos en torno a cada proyecto, se contratarán conceptos teóricos y se resolverán dudas que no hayan sido expuestas anteriormente. Al final de la clase cada grupo entregará sus fichas de proyecto.

Material necesario: PPT, fichas de proyecto e instrucciones del ejercicio [01]. La clase se desarrolla en el aula informática con el Software Adobe InDesgn.

\begin{tabular}{|l|l|l|}
\hline Número ficha: Sesión 4/ Ficha 8 & Fase metodológica: I + 0 & $\begin{array}{l}\text { Duración: } \\
20^{\prime \prime}\end{array}$ \\
\hline $\begin{array}{l}\text { Introducción y objetivos: en esta introducción revisaremos rápidamente } \\
\text { dónde nos quedamos el día anterior y fijaremos los objetivos de la sesión } \\
\text { que serán diseñar, por grupos, el producto multimedia que van a realizar } \\
\text { dentro de cada proyecto práctico de la asignatura. }\end{array}$ \\
\hline Material necesario: PPT.
\end{tabular}

\begin{tabular}{|l|l|l|}
\hline Número ficha: Sesión 4/ Ficha 9 & $\begin{array}{l}\text { Fase metodológica: Py + IA } \\
+P r+C t+P g\end{array}$ & $\begin{array}{l}\text { Duración: } \\
120^{\prime \prime}\end{array}$ \\
\hline
\end{tabular}

Jornadas de Formación e Innovación Docente del Profesorado | № 1 (2018) Esta obra se distribuye con la licencia Creative Commons Reconocimiento-NoComercial-SinObraDerivada $\quad 4.0$ Internacional (CC BY-NC-ND 4.0.) 
Proyecto + Iniciativa de los Alumnos + Contraste de la Teoría + Preguntas: esta sesión será de trabajo en grupo con ordenadores. Sobre las fichas del proyecto del borrador de las Especificaciones Funcionales de su proyecto los alumnos deben concretar que parte práctica [producto multimedia] van a realizar. Este trabajo incluye la definición del producto multimedia y un ejercicio práctico [01/Creación de un documento interactivo] con el software Adobe InDesign.

En ese tiempo el profesor estará pasando entre los grupos comentando sus ideas. En este tiempo, en función de la iniciativa de los alumnos en torno a cada proyecto, se contratarán conceptos teóricos y se resolverán dudas que no hayan sido expuestas anteriormente. Al final de la clase cada grupo entregará sus fichas de proyecto.

Material necesario: PPT, fichas de proyecto e instrucciones del ejercicio [01]. La clase se desarrolla en el aula informática con el Software Adobe InDesgn.

\begin{tabular}{|l|l|l|}
\hline Número ficha: Sesión 5/ Ficha 10 & Fase metodológica: I + O & $\begin{array}{l}\text { Duración: } \\
20 "\end{array}$ \\
\hline
\end{tabular}

Introducción y objetivos: en esta introducción revisaremos rápidamente dónde nos quedamos el día anterior y fijaremos los objetivos de la sesión que serán diseñar, por grupos, el producto multimedia que van a realizar dentro de cada proyecto práctico de la asignatura.

Material necesario: PPT.

\begin{tabular}{|l|l|l|}
\hline Número ficha: Sesión 5/ Ficha 11 & $\begin{array}{l}\text { Fase metodológica: } P y+I A+ \\
\operatorname{Pr}+C t+P g\end{array}$ & $\begin{array}{l}\text { Duración: } \\
120 "\end{array}$ \\
\hline
\end{tabular}

Proyecto + Iniciativa de los Alumnos + Contraste de la Teoría + Preguntas: esta sesión será de trabajo en grupo con ordenadores. Sobre las fichas del proyecto del borrador de las Especificaciones Funcionales de su proyecto los alumnos deben concretar que parte práctica [producto multimedia] van a realizar. Este trabajo incluye la definición del producto multimedia y un ejercicio práctico [02/Rollovers, creación de galerías e inserto de vídeos] con el software Adobe InDesign.

En ese tiempo el profesor estará pasando entre los grupos comentando sus ideas. En este tiempo, en función de la iniciativa de los alumnos en torno a cada proyecto, se contratarán conceptos teóricos y se resolverán dudas que no hayan sido expuestas anteriormente. Al final de la clase cada grupo entregará sus fichas de proyecto.

Material necesario: PPT, fichas de proyecto e instrucciones del ejercicio [02]. La clase se desarrolla en el aula informática con el Software Adobe InDesgn.

Número ficha: Sesión 6/ Ficha 12 Fase metodológica: । + 0

Duración: $20 "$ 
Introducción y objetivos: en esta introducción revisaremos rápidamente dónde nos quedamos el día anterior y fijaremos los objetivos de la sesión que serán diseñar, por grupos, el producto multimedia que van a realizar dentro de cada proyecto práctico de la asignatura.

Material necesario: PPT.

Número ficha: Sesión 6/ Ficha 13

Fase metodológica: $P y+\mid A+$ $\mathrm{Pr}+\mathrm{Ct}+\mathrm{Pg}$

Duración: $120 "$

Proyecto + Iniciativa de los Alumnos + Contraste de la Teoría + Preguntas: esta sesión será de trabajo en grupo con ordenadores. Sobre las fichas del proyecto del borrador de las Especificaciones Funcionales de su proyecto los alumnos deben concretar que parte práctica [producto multimedia] van a realizar. Este trabajo incluye la definición del producto multimedia y un ejercicio práctico [[02/Rollovers, creación de galerías e inserto de vídeos]] con el software Adobe InDesign.

En ese tiempo el profesor estará pasando entre los grupos comentando sus ideas. En este tiempo, en función de la iniciativa de los alumnos en torno a cada proyecto, se contratarán conceptos teóricos y se resolverán dudas que no hayan sido expuestas anteriormente. Al final de la clase cada grupo entregará sus fichas de proyecto.

Material necesario: PPT, fichas de proyecto e instrucciones del ejercicio [02]. La clase se desarrolla en el aula informática con el Software Adobe InDesgn.

Para intentar explorar y conocer los modelos mentales de los estudiantes (Rivero y Porlán, 2017) diseñamos un cuestionario inicial con las preguntas del mapa de conceptos que les pasamos el miércoles 17 de octubre de 2018 durante 30-40 minutos aproximadamente. El cuestionario tenía un encabezado donde se pedía un identificador anónimo para los alumnos, la fecha y esta frase: "Esto no es un examen, no cuenta para la nota y no tiene respuestas correctas ni erróneas. Responde lo que tú creas en función de tu intuición y tu experiencia." El resultado fue bastante bueno. Todos los alumnos contestaron a todas las preguntas, con distintos niveles de profundidad.

\section{Aplicación del CMD}

En resumen, las sesiones se desarrollaron como sigue: Siguiendo la secuencia de actividades prevista para la 
primera sesión realizamos la primera actividad de "Introducción y objetivos de la sesión”. ¿Cómo íbamos a alcanzar esos contenidos? Queríamos crear esas "circunstancias que conducen al aprendizaje relevante en terceras personas" (Finkel, 2008) y lo intentamos a través del análisis de un caso práctico. La actividad prevista era la "Práctica de visionado" que analiza el caso práctico de un proyecto transmedia concreto, "El universo transmedia de la serie La Peste". El visionado fue acompañado de una hoja de observación que repartí en papel antes de ver el vídeo, donde los alumnos fueron anotando, sobre un esquema dado, algunas de las ideas que se apuntaban en el vídeo para el debate posterior. Tuvimos que ver el vídeo varias veces porque el ritmo del montaje es muy rápido y hay mucha información condensada en muy poco tiempo. El debate fue muy interesante porque los alumnos veían los conceptos más abstractos contextualizados en el caso práctico y así les era mucho más fácil comprenderlos, una vez identificados. En los últimos minutos de la sesión, con una presentación en PowerPoint fuimos concretando esos conceptos teóricos y sistematizando la información para los alumnos. Fue una sesión muy satisfactoria porque los alumnos alcanzaron claramente los conceptos a través del visionado y el debate dirigido posterior. Se abordaron todos los conceptos que se habían planificado y las sensaciones fueron muy buenas.

En la segunda sesión, tras la "Introducción y objetivos", la segunda actividad era "Proyecto + Iniciativa de los Alumnos + Contraste de la Teoría + Preguntas". En la plataforma virtual y en papel en clase se repartieron una serie de fichas del proyecto entre los grupos. Los alumnos definieron antes de acabar la clase un primer borrador de las Especificaciones Funcionales de su proyecto. El trabajo en grupo se desarrolló muy bien, con muchas aportaciones de todos los grupos y con bastante claridad de ideas. De cada proyecto salieron diversos conceptos teóricos que pudimos ir contrastando y se resolvieron muchas dudas 
de la evolución del trabajo en grupo. Las sensaciones fueron buenas. El trabajo del día anterior estaba reciente y los alumnos aplicaron de forma creativa los conceptos y los modelos a sus propios proyectos.

Vamos a explicar las sesiones 3 y 4 conjuntamente, así como las 5 y 6, porque el modelo metodológico es el mismo ya que se trata de la misma sesión con un desdoble de la clase al realizarse en un aula informática con un número de plazas más limitada. En estas sesiones el trabajo en grupo era con ordenadores. Sobre las fichas del proyecto del borrador de las Especificaciones Funcionales de su proyecto realizado en la sesión anterior, los alumnos debían concretar que parte práctica [producto multimedia] van a realizar. Este trabajo incluye la definición del producto multimedia y un ejercicio práctico con el software Adobe InDesign. La evolución de esta práctica fue desigual. Por una parte, los alumnos se centraron en el manejo del software, lo cual fue muy positivo porque pudieron terminar todos ellos el ejercicio propuesto de manera mínimamente satisfactoria. Por otra parte, los alumnos tuvieron problemas al transferir los conceptos planteados en el ejercicio al proyecto de grupo y viceversa. Las buenas sensaciones respecto a las dos primeras sesiones empezaron a verse cuestionadas.

En las sesiones 5 y 6 sobre las fichas del proyecto del borrador de las Especificaciones Funcionales de su proyecto los alumnos debían terminar de concretar la parte práctica [producto multimedia] que van a realizar. Este trabajo incluía la definición del producto multimedia y un segundo ejercicio práctico con el software. Después de los problemas de las sesiones 3 y 4 en la transferencia de modelos a los casos concretos, introdujimos una variación en la práctica para contrastar si los conceptos estaban asentados. El resultado fue desigual. Al ser una práctica individual que luego había que poner en común, algunos alumnos demostraron tener dificultades con la aplicación de los modelos. Los nervios por la entrega de las prácticas 
al finalizar la sesión de dos horas evidenciaron más esas dudas conceptuales.

Haciendo un análisis y valoración de lo que ha funcionado mejor y de lo que no ha funcionado como esperábamos, tratando de entender las causas y consecuencias y buscando soluciones concretas para el futuro entendemos que el desarrollo de la sesión 1 fue muy bueno. La selección del vídeo es un acierto porque ofrece muchas posibilidades a nivel teórico. Para los alumnos fue mucho más fácil entender así los conceptos, con ejemplos claros y muy visuales. Aun así, las sesiones posteriores demostraron que los conceptos no estaban tan asentados como creíamos. La sesión 2, justo al día siguiente, fue muy productiva. Las fichas entregadas para el desarrollo del proyecto sirvieron para concretar muy bien las tareas a realizar. Todos comprendieron bien el encaje de los elementos dentro del proyecto y sus aportaciones creativas fueron muy coherentes y con muchas posibilidades de viabilidad. Las sesiones 3 y 4 fueron las más complejas. Nuestra idea de plantear un ejercicio "micro" individual con el software transferible al proyecto "macro" del grupo no funcionó como esperábamos. Los alumnos se centraron en resolver el ejercicio "micro" con el software, pero no comprendieron bien la transferencia al proyecto "macro". Esto nos hace pensar que los conceptos que creíamos asentados no lo estaban tanto. Es probable que la forma de plantear la práctica sea mejorable, haciendo más hincapié en su relación con el proyecto "macro". La distancia entre las sesiones, una semana, creemos que también es un factor a tener en cuenta. Puede resolverse con una tarea entre sesiones para casa. En las sesiones 5 y 6 la modificación en la práctica que realizamos vistos los resultados de las sesiones anteriores, ayudó a que los alumnos llegaran más o menos a los objetivos planteados. Esa misma modificación de la práctica, que evidenciaba que los conceptos 
no estaban muy bien afianzados, puso un poco nerviosos a bastantes alumnos durante el desarrollo de la práctica. Los trabajos en grupos son útiles para muchas cosas, pero también sirven para que muchos alumnos se oculten entre sus compañeros. Las prácticas individuales los sacan a la luz y muestran sus verdaderos conocimientos. Creemos que también por ese motivo, nuestra sensación general positiva a nivel de grupos fue un poco peor en las sesiones donde las prácticas eran en parte individuales.

Como comentamos anteriormente, preparamos un cuestionario inicial/final para poder explorar los modelos mentales de los estudiantes y hacer un seguimiento de su evolución en torno a los problemas clave. En el futuro debemos intentar definir preguntas más integradoras y evitar las respuestas SI o NO para que los alumnos puedan llevar a cabo respuestas más elaboradas e indagar mejor en sus modelos mentales. Después de pasar los cuestionarios, agrupamos las respuestas, las ordenamos, analizamos y establecimos conclusiones. Establecimos conclusiones e implicaciones didácticas que nos llevaron a ajustar el mapa de contenidos a la realidad de los estudiantes y a rediseñar una parte de la secuencia de actividades que ayudara a superar los obstáculos que habíamos detectado.

Los criterios que definimos para escalonar los modelos mentales de los estudiantes van desde una visión más simplista de la realidad hacia una visión progresivamente más compleja, relacionada y abstracta de la misma. Para presentar los resultados hemos unificado en una sola "escalera de aprendizaje" por pregunta los niveles iniciales y finales, poniendo en un color los porcentajes del cuestionario inicial y en otro los porcentajes del final. 


\section{CUESTIONARIO INICIAL / FINAL}

PREGUNTA 1. En un proyecto transmedia ¿qué medios pueden permitirnos alcanzara las audiencias y cumplir nuestros objetivos?
MODELOS DE RESPUESTAD.

Dependiendo de público objetivo y objetivos, cada medio hará lo que mejor sabe hacer.

\section{$\mathbf{7 0} \%$}

MODELOS DE RESPUESTAC. Depende del público objetivo.

$50 \%$

$\mathbf{2 5 \%}$

MODELOS DE RESPUESTA B.

Cualquier medio puede. MODELOS DE RESPUESTA A. No lo tiene claro.

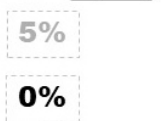

$35 \%$

$\mathbf{5 \%}$

Figura 3. Escalera de la pregunta 1 (cuestionarios inicial/final)

La pregunta 1 contiene un error general común que es el que se aprecia en los modelos de respuestas $B$ y $C$, que acumulan los mayores porcentajes de respuestas en el cuestionario inicial, 35\% y 50\% respectivamente. El balance del cuestionario final, en el que el 70\% de los encuestados están en el modelo de respuesta $D$, nos indica que los alumnos, en su mayoría, han asimilado bien estos conceptos. La actividad 2 de la sesión 1 del ciclo de mejora, y la actividad 3 de la sesión 1, fueron claves para entender la mejora en la respuesta a esta pregunta.

\section{CUESTIONARIO INICIAL / FINAL}

PREGUNTA 2. ¿Es conveniente crear todos los

MODELOS DE RESPUESTA D.

medios que utilicemos en el proyecto

No, hay medios creados,

transmedia?

ganadosy pagados.

$10 \%$

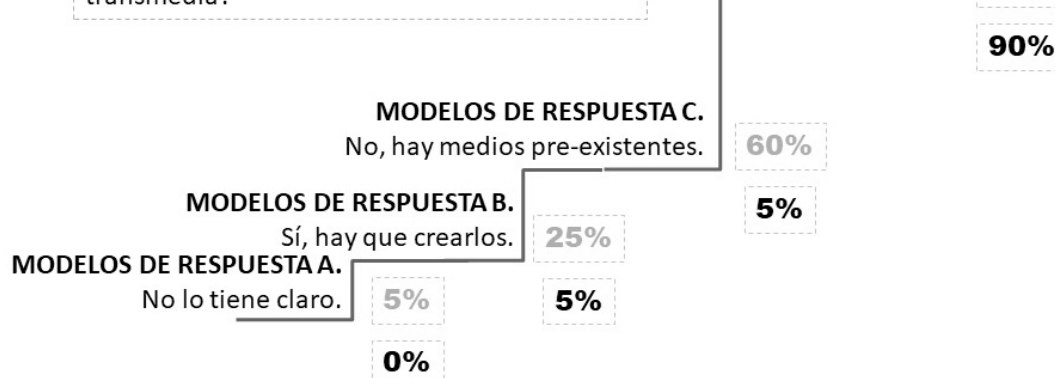

Figura 4. Escalera de la pregunta 2 (cuestionarios inicial/final)

Jornadas de Formación e Innovación Docente del Profesorado | № 1 (2018) Esta obra se distribuye con la licencia Creative Commons Reconocimiento-NoComercial-SinObraDerivada 
La pregunta 2 buscaba conceptos muy concretos; "medios creados, ganados y pagados". Estos conceptos se trataron repetidamente en todas las sesiones, lo que nos hace comprender que al final el $90 \%$ de los alumnos hayan llegado al nivel más complejo.

CUESTIONARIO INICIAL / FINAL

PREGUNTA 3. ¿Crees que la organización de la información y la experiencia del usuario están relacionadas? ¿Por qué?
MODELOS DE RESPUESTAD.

Sí, relaciona organización información con experiencia usuariosy diseño del $20 \%$ proyecto.

$90 \%$

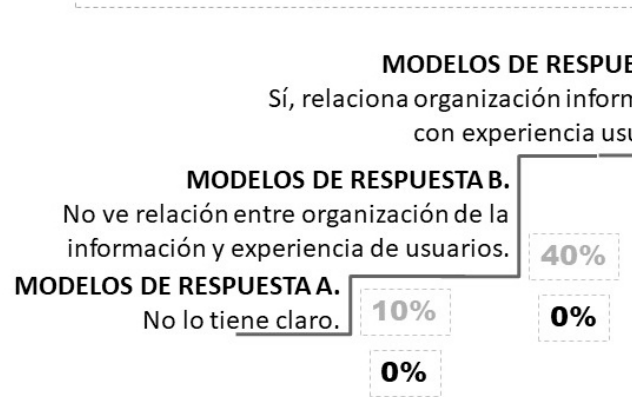

Figura 5. Escalera de la pregunta 3 (cuestionarios inicial/final)

La pregunta 3, más compleja, cruza conceptos como "organización de la información, experiencia del usuario y diseño del proyecto". Los alumnos de partida no tienden a asociar la "organización de la información" con la "experiencia del usuario", o si llegan a hacerlo no vinculan esa relación con el "diseño del proyecto". Por eso nos encontramos con un $40 \%$ y un $30 \%$ de las respuestas entre esos modelos de respuesta. Al estar trabajando constantemente sobre el proyecto global y tener que plantearse y cuestionarse los alumnos todos los pasos y decisiones que implica su diseño, es comprensible que al final un $90 \%$ de ellos hayan alcanzado el nivel más complejo de respuesta. A pesar de ello, hay un $10 \%$ de alumnos que siguen sin completar el razonamiento que buscábamos y sus respuestas siguen estando incompletas. 
CUESTIONARIO INICIAL / FINAL

PREGUNTA 4. ¿Cómo crees que afecta la implicación del usuario en el éxito de un proyecto transmedia?
MODELOS DE RESPUESTAD.

Relaciona la implicación del usuario con el éxito del proyecto pero entiende que el éxito puede ser el cumplimiento de otros objetivos.

$20 \%$

$\mathbf{8 0} \%$
Vincula totalmente la implicación del usuario con el éxito del proyecto.

MODELOS DE RESPUESTA B.

No ve relación entre implicación del usuario y éxito del proyecto. MODELOS DE RESPUESTAA.

No lo tiene claro.

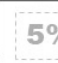

$\mathbf{0 \%}$

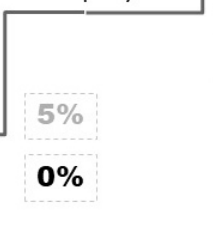

$\mathbf{2 0} \%$

Figura 6. Escalera de la pregunta 4 (cuestionarios inicial/final)

La pregunta 4 abarca el concepto de "éxito del proyecto". Para muchos alumnos el concepto de "éxito" siempre está asociado a la participación del usuario y muchos de ellos se resisten a aceptar que haya otros indicadores de éxito: Por ello encontramos 70\% de los alumnos en el modelo C. Al finalizar el ciclo, y tras plantear sus proyectos, la mayor parte de los alumnos, un $80 \%$, llegan al modelo D de respuesta más compleja, mientras que un $20 \%$ no consigue alcanzarlo.

Jornadas de Formación e Innovación Docente del Profesorado I № 1 (2018) Esta obra se distribuye con la licencia Creative Commons 
Tabla 1. Cuadro de evolución por estudiantes (muestra de alumnos)

\begin{tabular}{|c|c|c|c|c|c|c|c|c|c|c|c|c|}
\hline & \multicolumn{3}{|c|}{ PREGUNTA 1} & \multicolumn{3}{|c|}{ PREGUNTA 2} & \multicolumn{3}{|c|}{ PREGUNTA 3} & \multicolumn{3}{|c|}{ PREGUNTA 4} \\
\hline & $\mathbf{I}$ & $\mathbf{F}$ & $\mathbf{P}$ & I & $\mathbf{F}$ & $\mathbf{P}$ & $\mathbf{I}$ & $\mathbf{F}$ & $\mathbf{P}$ & $\mathbf{I}$ & $\mathbf{F}$ & $\mathbf{P}$ \\
\hline ESTUDIANTE 1 & B & D & $\uparrow \uparrow$ & $C$ & D & $\uparrow$ & B & D & $\uparrow \uparrow$ & A & C & $\uparrow \uparrow$ \\
\hline ESTUDIANTE 2 & A & C & $\uparrow \uparrow$ & C & $\mathrm{D}$ & $\uparrow$ & C & D & $\uparrow$ & C & D & $\uparrow \uparrow$ \\
\hline ESTUDIANTE 3 & B & D & $\uparrow \uparrow$ & C & D & $\uparrow$ & C & D & $\uparrow$ & C & D & $\uparrow \uparrow$ \\
\hline ESTUDIANTE 4 & A & B & $\uparrow$ & $A$ & B & $\uparrow$ & B & C & $\uparrow$ & C & D & $\uparrow \uparrow$ \\
\hline ESTUDIANTE 5 & D & D & $\rightarrow$ & B & D & $\uparrow \uparrow$ & B & D & $\uparrow \uparrow$ & C & D & $\uparrow \uparrow$ \\
\hline ESTUDIANTE 6 & B & D & $\uparrow \uparrow$ & B & C & $\uparrow$ & C & D & $\uparrow$ & C & D & $\uparrow \uparrow$ \\
\hline ESTUDIANTE 7 & B & D & $\uparrow \uparrow$ & B & C & $\uparrow$ & C & D & $\uparrow$ & C & D & $\uparrow \uparrow$ \\
\hline ESTUDIANTE 8 & B & D & $\uparrow \uparrow$ & $C$ & D & $\uparrow$ & C & D & $\uparrow$ & C & D & $\uparrow \uparrow$ \\
\hline ESTUDIANTE 9 & B & D & $\uparrow \uparrow$ & B & C & $\uparrow$ & C & D & $\uparrow$ & C & D & $\uparrow \uparrow$ \\
\hline$\cdots$ & $\ldots$ & $\ldots$ & $\ldots$ & $\ldots$ & $\ldots$ & $\ldots$ & $\ldots$ & $\ldots$ & $\ldots$ & $\ldots$ & $\ldots$ & $\ldots$ \\
\hline
\end{tabular}

\section{Evaluación del CMD}

Estamos bastante satisfechos con el resultado del ciclo de mejora. La elaboración de los modelos metodológicos nos ha ayudado mucho en la planificación de las clases. Debemos mejorar la gestión de los tiempos, lo cual nos produce retrasos en el cumplimiento de algunos objetivos, que solemos compensarlos en la siguiente sesión. La participación de los alumnos es mejorable, pero el clima parece bueno y creemos que se puede subir el nivel de participación. Nos quedamos con muchas cosas positivas: La eficacia del visionado de ejemplos y su análisis para sacar los conceptos; sacar del caso práctico concreto, los conceptos y no al revés; la mezcla de ejercicio individual y posterior puesta en común. Debemos mejorar la relación entre las prácticas "micro" con los proyectos "macro". Cuando los alumnos entendieron la transferencia entre unas prácticas y otras fue muy productivo. Pasar lista y sobre todo recoger el trabajo al final de la sesión también han resultado 
muy bien porque han mantenido el nivel de concentración de los alumnos muy alto.

Hemos definido algunos principios didácticos que han guiado la experiencia y que deben permanecer en el futuro:

a) En cuanto al aprendizaje de los/as alumnos/as: Una idea fundamental es que este ciclo nos ha hecho reflexionar sobre toda la actividad docente; desde la metodología, a la forma de evaluación, pasando por los contenidos. Incluso nos ha hecho replantearnos la relación con los alumnos. Y eso, en sí mismo, ya es muy positivo.

Hemos profundizado en la idea de que el aprendizaje es una interacción constructiva entre lo que yo ya sé y lo que me ayudan a conocer. Eso nos ha ayudado a mejorar la forma de trabajar sobre las ideas y los modelos mentales de los estudiantes de una forma más analítica, para centrar luego la enseñanza en ellos.

b) En cuanto a los contenidos de enseñanza: Las "Preguntas encadenadas" nos han llevado a enlazar los contenidos y para la selección de los contenidos esenciales fueron muy importantes los mapas de contenidos. El resultado fue transformar el temario de una sucesión lineal de contenidos "todos importantes", a una matriz de contenidos, no necesariamente lineal, más bien cíclica, interrelacionada, hipertextual, pero jerarquizada y articulada en torno a esas preguntas básicas.

c) En cuanto a la metodología: Ha sido clave el diseño del modelo, la definición de las preguntas y la secuencia de actividades. Una secuencia de actividades detallada, que responda a una metodología bien diseñada ha resultado enormemente útil y ha aportado mucha seguridad en las clases. 
d) En cuanto a la evaluación: Nos parece la parte más complicada y la más delicada de todo el proceso de cambio; evaluación de nuestra docencia, contenidos, metodología y sistema de evaluación. El trabajo que hemos realizado con el análisis de nuestro modelo metodológico, los cuestionarios previos y finales, las escaleras de aprendizaje y los mapas de contenidos, nos ha dado una visión más amplia de la importancia de nuestra propia evaluación y la necesidad de que tenga una proyección clara en todo el proceso docente.

A partir de estas reflexiones vamos a intentar extraer nuestros Principios Didácticos Personales en este momento o nuestro Modelo Didáctico Personal de referencia:

I. Reflexionar sobre qué queremos que aprendan los alumnos en cada una de nuestras asignaturas. Enfrentando eso con el programa de la asignatura.

II. Definir nuestro modelo metodológico posible de cada asignatura.

III. Trabajar con "problemas" como vehículos para que los alumnos tomen la iniciativa y los resuelvan con sus hipótesis.

IV. Replantear los contenidos de las asignaturas para que sirvan como andamiaje para que los alumnos construyan sobre ellos.

V. Utilizar preguntas encadenadas para guiar a los alumnos en todo el proceso.

VI. Diseñar los contenidos de manera que el alumno realice la transferencia del pensamiento concreto al pensamiento formal. Para que vaya del aprendizaje situado a la transferencia a modelos. VII. Profundizar en el conocimiento de los modelos mentales de los alumnos, detectando los obstáculos y ajustando los contenidos de la asignatura. 
VIII. Configurar dinámicas de trabajo que vayan del trabajo individual al trabajo en grupo.

IX. Establecer una evaluación continua, basada en el compromiso y el trabajo de alumnos y profesor. Planteando una fórmula presencial y otra no presencial. Evaluándonos todos y evaluándolo todo.

X. Hacer todo esto con empatía. Tratando al alumno como persona. Mostrarnos como referentes para ellos.

\section{Referencias bibliográficas.}

Bain, K. (2007): Lo que hacen los mejores profesores universitarios, Valencia, Universidad de Valencia.

Finkel, D. (2008): Dar clase con la boca cerrada, Valencia, Universidad de Valencia.

Porlán, R. (Coord.) (2017): Enseñanza Universitaria. Cómo mejorarla, Madrid, Ediciones Morata.

Vergara, J.J. (2017): Aprendo porque quiero. El Aprendizaje Basado en Proyectos (ABP) paso a paso, Madrid, Editorial SM.

Jornadas de Formación e Innovación Docente del Profesorado | № 1 (2018) Esta obra se distribuye con la licencia Creative Commons 